\title{
GAIA Gestational Diabetes Mellitus Level of Diagnostic Certainty Terminology
}

National Cancer Institute

\section{Source}

National Cancer Institute. GAIA Gestational Diabetes Mellitus Level of Diagnostic

Certainty Terminology. NCI Thesaurus. Code C128709.

A subset of terminology related to gestational diabetes mellitus, developed by the Global Alignment of Immunization safety Assessment in pregnancy consortium to aid in monitoring and improving fetal and maternal outcomes. 